\title{
Co-expression network analysis of IncRNAs and mRNAs in OPTN-silenced cells
}

\author{
LIN JIN, YUANYUAN LI, XINRONG ZHOU and HUIPING YUAN \\ Department of Ophthalmology, The Second Affiliated Hospital of Harbin Medical University, \\ Harbin, Heilongjiang 150081, P.R. China
}

Received January 29, 2016; Accepted November 30, 2017

DOI: $10.3892 / \mathrm{ijmm} .2017 .3313$

\begin{abstract}
The aim of the present study was to reveal the role of long noncoding RNAs (IncRNAs) in the regulation of the pathogenesis of optineurin (OPTN)-silenced cells. The microarray data set GSE12452 was re-annotated using the non-coding RNA function annotation server to identify differentially expressed lncRNAs. Weighted correlation network analysis was used to construct an lncRNA-lncRNA co-expression network and identify co-expression modules. Three OPTN small interfering RNAs (siRNAs) were transiently transfected into HeLa cells. Reverse transcription-quantitative polymerase chain reaction (RT-qPCR) and western blot analysis were used to detect OPTN expression and select the most effective OPTN siRNA to construct stably transfected cells. RT-qPCR was used to quantify the identified lncRNAs in the OPTN-silenced cells. The potential functions of these modules were explored by the functional enrichment of the corresponding co-expressed genes. A total of 3,495 lncRNAs were re-annotated. Of these, matrix metalloprotease 12 and RP11-169D4.1 were upregulated, and RP1-212P9.2 was downregulated. The results of the RT-qPCR analysis of RP1-212P9.2 and RP11-169D4.1 were consistent with the re-annotated data in the OPTN-silenced cells. Gene Ontology analyses indicated that the biological functions of the mRNAs co-expressed with these lncRNAs were associated with gene product regulation, and neuronal migration, polarity and differentiation. In addition, Kyoto Encyclopedia of Genes and Genomes analysis indicated that the two validated IncRNAs were associated with the transforming growth factor- $\beta$ signaling pathway and the apoptosis pathway, respectively. In conclusion, the abnormal lncRNAs identified in OPTN-silenced cells indicate that lncRNAs may contribute to the molecular pathogenesis of OPTN-associated diseases.
\end{abstract}

Correspondence to: Dr Huiping Yuan, Department of Ophthalmology, The Second Affiliated Hospital of Harbin Medical University, 246 Xuefu Road, Harbin, Heilongjiang 150081, P.R. China

E-mail: yuanhp2013@126.com

Key words: optineurin, long noncoding RNAs, transforming growth factor- $\beta$, apoptosis, glaucoma

\section{Introduction}

Primary open-angle glaucoma (POAG) is characterized by degenerative changes in the optic nerve and retinal ganglion cells (RGCs) and leads to progressive visual field loss with or without elevated intraocular pressure (IOP) (1). Numerous studies have demonstrated that genetic factors serve an important role in POAG. It has been found that mutations in the optineurin (OPTN) gene may be associated with RGC degeneration and increase susceptibility to the development of POAG $(2,3)$. Furthermore, studies have indicated that OPTN is important in various physiological processes, including the nuclear factor- $\mathrm{\kappa B}$ pathway, vesicle trafficking, protein secretion and the control of cell division $(4,5)$. These functions depend on the precise subcellular localization of OPTN, its post-translational modifications and interactions with binding partners, including Rab8, huntingtin and myosin VI $(6,7)$. However, how these diverse functions of OPTN are integrated into a comprehensive network remains unclear.

Long noncoding RNAs (lncRNAs) are a class of RNA molecules $>200$ nucleotides in length that structurally resemble mRNAs but do not encode proteins (8). Studies have shown that IncRNAs are often interspersed between, or overlap with diverse coding and non-coding transcripts (9). The extensive functions of lncRNAs include precise subcellular localization, high-order chromosomal dynamics, telomere biology and regulation of the expression of neighboring protein-coding genes (10-12). Highly sensitive genome tiling arrays have demonstrated that the ability of IncRNAs to regulate protein-coding genes may serve important roles in various human diseases, including cardiovascular diseases, cancer and neurological diseases $(13,14)$. OPTN has been identified as a gene that is associated with hereditary neurological diseases $(2,15)$. In addition, IncRNA as a key regulator rarely exerts a biological function alone and may act synergistically $(16,17)$. Thus, the analysis of OPTN-associated lncRNAs by constructing an lncRNA-lncRNA co-expression network for OPTN and identifying lncRNA co-expression modules may potentially improve our understanding of the complex molecular mechanisms associated with the pathogenesis of OPTN-associated diseases.

To investigate the underlying role of lncRNAs in the regulation of OPTN function, OPTN-silencing microarray data was re-annotated in the present study to identify differen- 
tially expressed lncRNAs and the potential functions of these lncRNAs were explored by functional enrichment analysis of the corresponding co-expressed genes.

\section{Materials and methods}

Microarray. The dataset GSE6819 was downloaded from the Gene Expression Omnibus (GEO) database (http://www. ncbi.nlm.nih.gov/geo/query/acc.cgi?acc=GSE6819). This microarray dataset was designed to investigate the comprehensive molecular mechanisms by which OPTN mediates, and identified genome-wide molecular changes upon OPTN silencing in HeLa cells by microarray technology (18). It comprises three samples treated with small interfering RNA (siRNA) specific for OPTN mRNA and three samples treated with water (mock experiment) using the Human Genome U133 (HG-U133) Plus 2.0 array (Affymetrix; Thermo Fisher Scientific, Inc., Waltham, MA, USA).

Functional re-annotation of lncRNAs. The non-coding RNA function annotation server (ncFANs) was used to re-annotate the probes on the HG-U133 Plus 2.0 array (19). Differentially expressed lncRNAs were selected using the fold-change method with an absolute $\log _{2}$ fold-change threshold of $>1$. The fold-change values for each probe were calculated. Each probe was then converted to its Entrez Gene ID. If one gene matched more than one probe, its expression value was computed by averaging the expression values of all corresponding probes.

Construction of a lncRNA-lncRNA co-expression network and detection of co-expression modules. Pearson's correlation coefficients (PCCs) between expressed values of each lncRNA in all samples were calculated using the weighted correlation network analysis (WGCNA) R software package, which is a comprehensive collection of $\mathrm{R}$ functions for performing various aspects of weighted correlation network analysis (20). The package includes functions for network construction, module detection, gene selection and calculations of topological properties. The co-expressed lncRNA pairs with $\mathrm{P}<0.01$ and absolute PCC $>0.75$ were selected. Furthermore, IncRNA-lncRNA co-expression modules were also detected using this package. The average absolute fold-change values of IncRNAs for all modules were then calculated. The top three differential modules with the greatest average absolute fold-change values were selected.

Cell culture and knockdown of OPTN. The HeLa cell line (American Type Culture Collection, Manassas, VA, USA) was cultured in Dulbecco's modified Eagle's medium (Hyclone, Logan, UT, USA) containing 10\% fetal bovine serum (Gibco-BRL; Thermo Fisher Scientifc, Grand Island, $\mathrm{NY}, \mathrm{USA}), 20 \mu \mathrm{g} / \mathrm{ml}$ penicillin and $20 \mu \mathrm{g} / \mathrm{ml}$ streptomycin at $37^{\circ} \mathrm{C}$ in a humidified atmosphere with $5 \% \mathrm{CO}_{2}$. The day prior to transfection, the cells were plated in a 6 -well plate $(10,000$ cells/well) in an appropriate volume of growth medium without antibiotics such that they were 30-50\% confluent at the time of transfection. siRNAs were transfected using $10 \mu \mathrm{l} /$ well X-tremeGENE siRNA Transfection reagent (Roche Diagnostics, Basel, Switzerland) at $10 \mathrm{nM}$ final concentration, and the cells were harvested after $48 \mathrm{~h}$.
Table I. siRNA targeting sequences of OPTN.

Genes $\quad$ Sequences (5'-3')

siOPTN-1 (si-h-optineurin-1) siOPTN-2 (si-h-optineurin-2)

GAAGCCATGAAGCTAAATA siOPTN-3 (si-h-optineurin-3)

CTGCAGCTCAAGCTGAACT

CCATCAGAGTTGAATGAAA

siRNA, small interfering RNA; OPTN, optineurin

The siRNA oligonucleotides were synthesized by Guangzhou Ribobio Co., Ltd. (Guangzhou, China). The sequences are presented in Table I.

$R N A$ isolation and reverse transcription-quantitative polymerase chain reaction ( $R T-q P C R)$. Total RNA was isolated from cultured HeLa cells using a High Pure RNA Isolation kit (Roche Diagnostics). Complementary DNA was synthesized from $1 \mu \mathrm{g}$ total RNA using the High Capacity cDNA Reverse Transcription kit (Applied Biosystems; Thermo Fisher Scientific, Inc.). All RT-qPCR experiments were run in triplicate as singleplex reactions on the 7500 Fast Real-Time PCR system (Applied Biosystems; Thermo Fisher Scientific, Inc.) using SYBR-Green (Roche Diagnostics), according to the manufacturer's instructions. The RT-qPCR conditions involved a denaturation step $\left(95^{\circ} \mathrm{C}\right.$ for $\left.10 \mathrm{~min}\right)$, and amplification and quantification were repeated 40 times $\left(95^{\circ} \mathrm{C}\right.$ for $15 \mathrm{sec}, 56^{\circ} \mathrm{C}$ for $15 \mathrm{sec}$ and $72^{\circ} \mathrm{C}$ for $30 \mathrm{sec}$, respectively). The relative gene expression levels were quantified based on the quantification cycle $(\mathrm{Cq})$ and normalized to the reference gene glyceraldehyde 3-phosphate dehydrogenase (GAPDH). The $\Delta \Delta \mathrm{Cq}$ method was used for relative quantification of gene expression, according to MIQE guidelines (21). All primers used in the reactions are presented in Table II.

Protein isolation and immunoblotting. Cells were lysed in radioimmunoprecipitation assay buffer supplemented with protease inhibitor cocktail (Biotool LLC, Houston, TX, USA) for $30 \mathrm{~min}$ on ice to extract the total protein. Protein concentrations were determined using a bicinchoninic acid protein assay (OriGene Technologies, Inc., Beijing, China). The protein samples were boiled in $1 \mathrm{X}$ sodium dodecyl sulfate buffer for $10 \mathrm{~min}$. Equal amounts of proteins (50 $\mu \mathrm{g} /$ lane) were subjected to $10 \%$ sodium dodecyl sulfate-polyacrylamide gel electrophoresis and transferred onto polyvinylidene fluoride membranes. The membranes were blocked with 5\% skimmed milk for $1 \mathrm{~h}$ at room temperature, probed with antibodies and visualized using an enhanced chemiluminescence reagent (OriGene Technologies, Inc.). Western blot analysis was performed to detect the OPTN protein, and GAPDH was used as the internal reference. Primary antibodies were anti-OPTN (1:400; sc-271549) and anti-GAPDH (1:5,000; sc-47724) (both from Santa Cruz Biotechnology, Inc., Dallas, TX, USA) (incubation at $4^{\circ} \mathrm{C}$ overnight). The secondary antibody was rabbit anti-mouse alkaline phosphatase-IgG (1:500; TA130002; OriGene Technologies, Inc.) (incubation for $1 \mathrm{~h}$ at room temperature). The protein bands were quantified using a Bio-Rad ChemiDoc ${ }^{\mathrm{TM}}$ EQ densitometer and Bio-Rad Quantity 
Table II. Primer and probe sequences used in reverse transcription-quantitative polymerase chain reaction.

\begin{tabular}{lll}
\hline Genes & \multicolumn{1}{c}{ Forward primers (5'-3') } & Reverse primers (5'-3') \\
\hline OPTN & AAAGGCCCGGAGACTGTTG & CATGTTCGTCATGGGTGTGAA \\
GAPDH & GTGTGCCGGTGCAAATACAC & CCTTTCAAGGGCCTGACACTT \\
RP1-212P9.2 & GGCATGGACTGTGGTCATGAG & CGTGCAGAGGCCCAGAGA \\
RP11-169D4.1 & TGGTCAGGCTGGTCTTGAACT & CAGGCCACGGTCACTCATATT \\
\hline
\end{tabular}

OPTN, optineurin; GAPDH, glyceraldehyde 3-phosphate dehydrogenase.

One software 4.62 (Bio-Rad Laboratories, Inc., Hercules, CA, USA).

Identification of co-expressed genes and functional enrichment. To explore the potential functions of the differentially expressed lncRNAs, co-expressed genes for the lncRNAs in the selected co-expressing modules were detected. To do this, the PCCs between each differentially expressed lncRNA and all genes across all samples were calculated. The genes with a strict cut-off (PCC $>0.9$ or $<-0.9$ ) were identified as co-expressed genes. Gene Ontology (GO) analysis was then conducted and gene sets were mapped to Kyoto Encyclopedia of Genes and Genomes (KEGG) pathways to identify the potential biological functions of the genes co-expressed with the lncRNAs using the Database for Annotation, Visualization and Integrated Discovery (DAVID, version 6.7) (22).

Statistical analysis. Data are presented as the mean \pm standard error of the mean, unless otherwise stated. Differences between the groups were analyzed using analysis of variance followed by Dunnett's test. The analysis was performed with SPSS 18.0 software (SPSS, Inc., Chicago, IL, USA). All tests were two-sided. $\mathrm{P}<0.05$ was considered to indicate a statistically significant result.

\section{Results}

Re-annotated microarray data. A total of 3,495 lncRNAs were re-annotated. Three lncRNAs with high fold-change values in the OPTN-silenced cells as compared with normal cells in the microarray were identified. These were matrix metalloprotease 12 (MMP12; upregulated), RP11-169D4.1 (upregulated) and RP1-212P9.2 (downregulated).

LncRNA-lncRNA co-expression network and co-expression modules. The lncRNA-lncRNA co-expression network was constructed using the WGCNA R software package. In this network, nodes represented IncRNAs and two nodes were connected if the PCC between the expression values of these two lncRNAs was $>0.7$ or $<-0.7$ and $P<0.01$. There were 3,495 nodes and 49,836 edges in this network (Fig. 1A). In addition, WGCNA analysis also detected 74 modules in this network. The three modules with the greatest average absolute fold-change values are presented in Fig. 1B. In module 3 , RP1-212P9.2 was the most strongly downregulated (blue ellipse in Fig. 1B).
Validation of OPTN knockdown. Three OPTN siRNA-expressing sequences (siOPTN-1, siOPTN-2 and siOPTN-3) and one negative control (siOPTN-NC) were transiently transfected into HeLa cells. The knockdown of OPTN by the three siRNAs was assayed using RT-qPCR. As shown in Fig. 2A, the cells transfected with siOPTN-1, siOPTN-2 and siOPTN-3 exhibited 50.12, 40.37 and $34.74 \%$ OPTN expression relative to the untransfected cells, respectively, all of which were found to be significant reductions as compared with the control $(\mathrm{P}<0.01 ; \mathrm{n}=3)$. OPTN levels in the cells transfected with siOPTN-NC were not observed to be significantly different from those in the untransfected cells $(86.01 \% ; \mathrm{P}>0.05 ; \mathrm{n}=3)$, indicating that the transfection itself did not affect OPTN expression. Western blot analysis was used to validate the RT-qPCR results (Fig. 2B and C). As shown in Fig. 2C, siOPTN-2 and -3 significantly inhibited OPTN protein expression $(\mathrm{P}<0.05 ; \mathrm{n}=3)$. Therefore, siOPTN-2 and -3 were used to construct stably transfected cell lines, which were used in the following experiments.

Validation of lncRNAs with RT-qPCR. To verify the results obtained with the re-annotated data, RT-qPCR assays were performed to detect the differences of IncRNA expression between cells transfected with siOPTN-2 and -3 and untransfected cells (Fig. 3). RP1-212P9.2 was significantly downregulated $(\mathrm{P}<0.01)$ and RP11-169D4.1 was significantly upregulated $(\mathrm{P}<0.01)$, which was consistent with the re-annotated data.

Detection of lncRNA functions. Gene enrichment provides a means of evaluating gene and gene product enrichment according to function, to facilitate the identification of GOs with particular relevance $(23,24)$. In the present study, it was identified that the RP1-212P9.2 and RP11-169D4.1 co-expressed mRNAs were most significantly associated with 'negative regulation of gene expression' and 'microtubule cytoskeleton organization' (ontology, biological process), respectively (Fig. 4). To illustrate the potential biological functions of IncRNAs in the modules, a co-expression network was constructed for the co-expressed coding genes of RP1-212P9.2 and RP11-169D4.1 and these coding genes were mapped to KEGG pathways using KEGG data mapping tools. The pathways comprised apoptosis (path, 04210), oxidative phosphorylation (path, 00190), axon guidance (path, 04360), ubiquitin-mediated proteolysis (path, 04120), cell cycle (path,04110), lysosome (path,04142), endocytosis (path,04144), transforming growth factor (TGF)- $\beta$ signaling pathway (path, 04350) and neurotropin signaling pathway (path, 04722). 

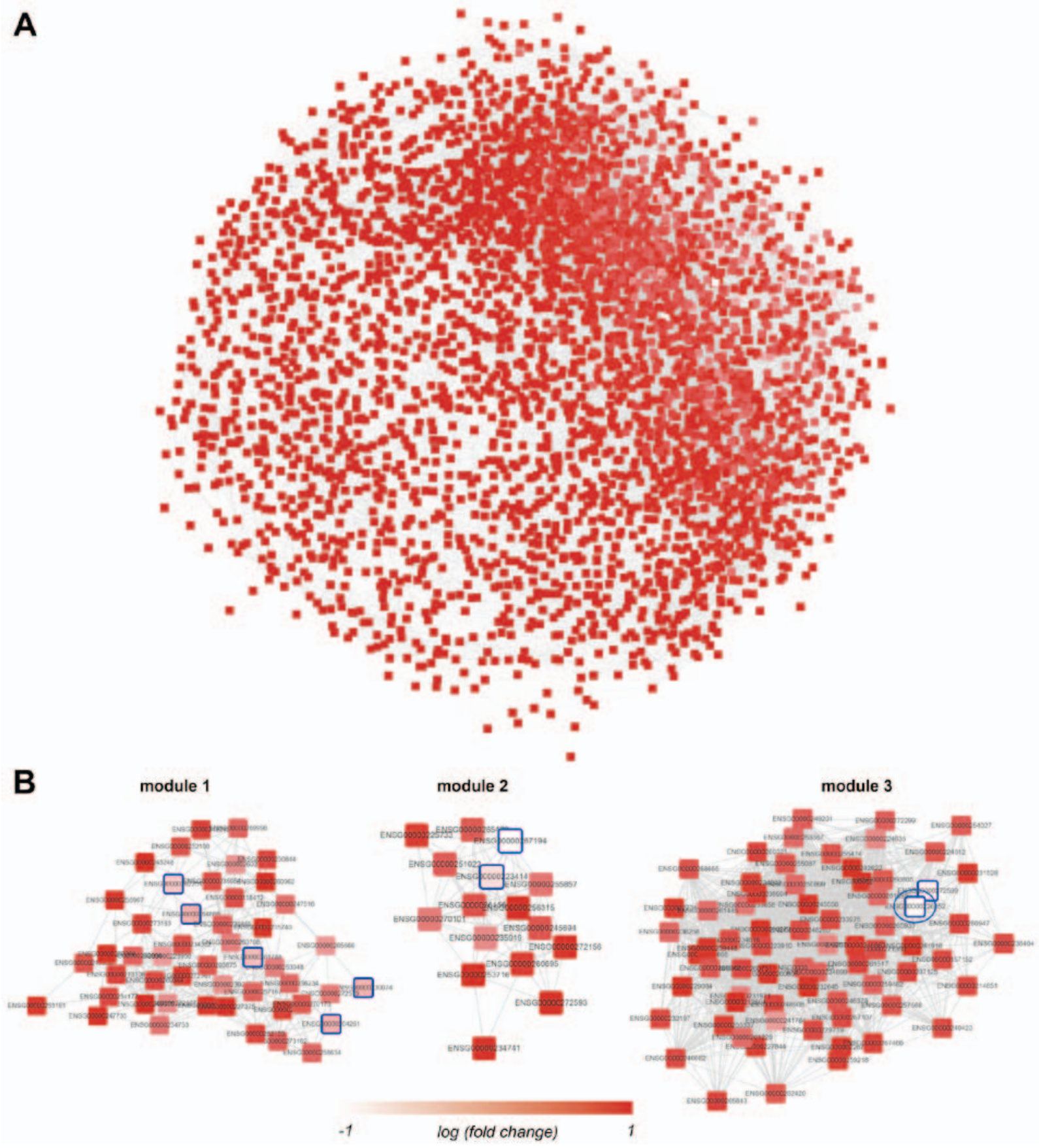

Figure 1. Construction of the IncRNA-lncRNA co-expression network and co-expression modules. (A) The co-expressed lncRNA pairs with PCC $>0.75$ or $<0.75$ and $\mathrm{P}<0.01$ were selected. (B) The top three differential modules were selected by calculating the average absolute fold-change values of lncRNAs for all modules (PCC $>0.9$ or $<-0.9$ ). The lncRNAs with a blue border are downregulated lncRNAs. IncRNA, long noncoding RNA; PCC, Pearson's correlation coefficient.

Among these pathways, the TGF- $\beta$ signaling pathway and apoptosis pathway are associated with basic biological processes of neural functions. The red genes represent co-expressed genes of the annotated lncRNAs (Fig. 5A-b and B-b).

\section{Discussion}

As the sensitivity of genome tiling arrays has increased, the concept of the functional genome has been revised to encompass an abundance of newly discovered lncRNA transcripts. lncRNAs are widely expressed in the mammalian nervous system and numerous lncRNAs are likely to serve critical roles in neuronal development and activity (13). However, the changes of IncRNA expression associated with ocular disorders are just beginning to be elucidated (25).

Previous studies have shown that disturbing the homeostasis of OPTN by overexpression or knockdown results in adverse consequences on cellular function, leading to the progression of neurodegenerative diseases including amyloid lateral sclerosis, Paget's disease of bone and POAG $(2,15,26)$. Glaucoma is the second most common cause of blindness in humans, and the most common form of this disease is POAG, which is caused by the irreversible progressive degeneration of RGCs (27). To investigate the molecular mechanisms and 

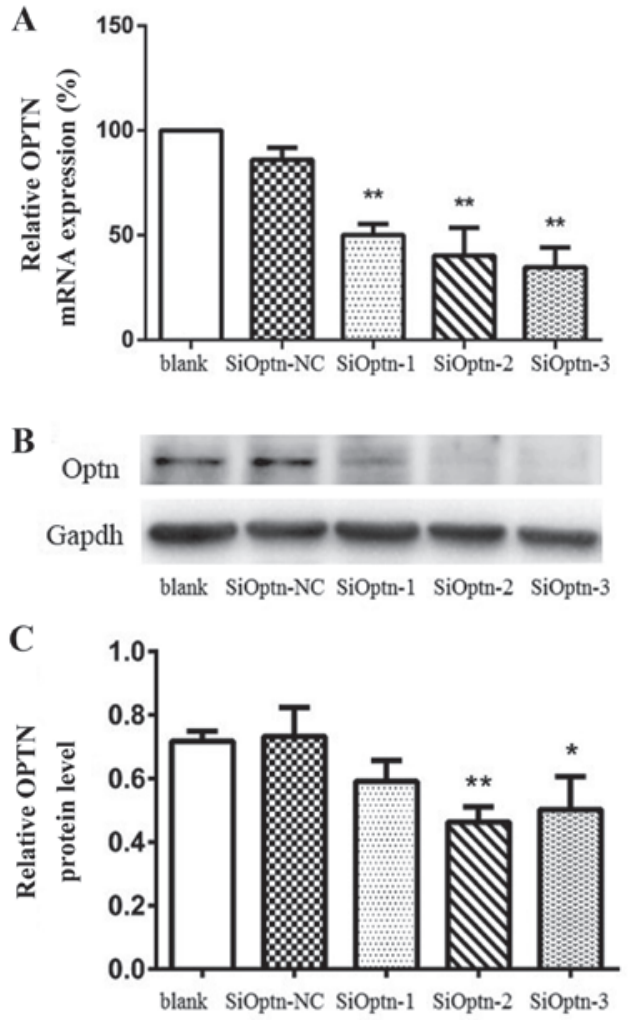

Figure 2. Effects of OPTN siRNA on OPTN expression in transfected cells. (A) Reverse transcription-quantitative polymerase chain reaction was used to measure OPTN mRNA expression. Mock transfected cells were set as $100 \%$. siOPTN-1, siOPTN-2 and siOPTN-3 were effective in inhibiting OPTN mRNA expression $(\mathrm{P}<0.05 ; \mathrm{n}=3)$. GAPDH served as the endogenous control. (B) Western blot analysis was used to measure the amount of OPTN protein in siOPTN-1, siOPTN-2, siOPTN-3 and siOPTN-NC transiently transfected and untransfected HeLa cells. (C) siOPTN-2 and siOPTN-3 were effective in inhibiting OPTN protein expression $(\mathrm{P}<0.05 ; \mathrm{n}=3)$. GAPDH served as the loading control $\left({ }^{*} \mathrm{P}<0.05\right.$ and ${ }^{* *} \mathrm{P}<0.01$ vs. the blank control; $\mathrm{n}=3$ ). OPTN, optineurin; siRNA, small interfering RNA.
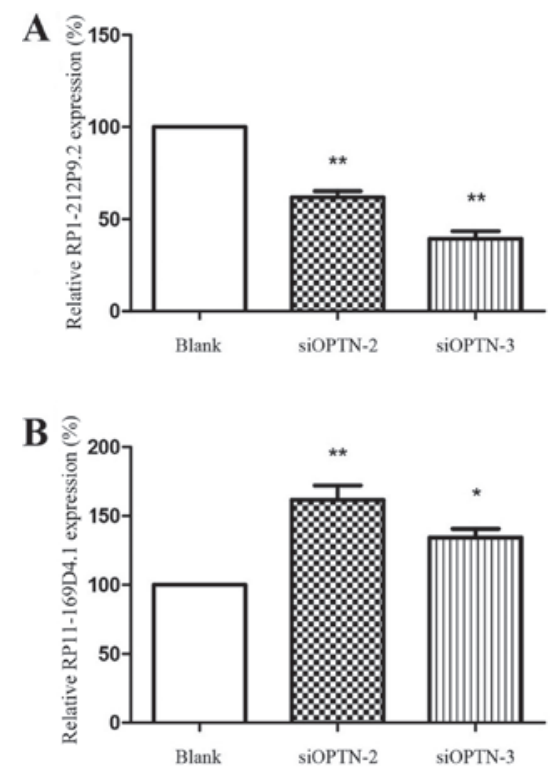

Figure 3. Relative expression levels of differentially expressed long noncoding RNAs in cells transfected with OPTN small interfering RNA (A) RP1-212P9.2 was downregulated (P<0.01; n=3). (B) RP11-169D4.1 was upregulated $(\mathrm{P}<0.01 ; \mathrm{n}=3)$. Relative expression levels were normalized to GAPDH by quantitative polymerase chain reaction analysis $\left({ }^{*} \mathrm{P}<0.05\right.$ and ${ }^{* *} \mathrm{P}<0.01$ vs. blank; $\mathrm{n}=3$ ). OPTN, optineurin. signal pathways of OPTN in POAG, Weisschuh et al (18) used RNA interference technology to silence the expression of OPTN in HeLa cells, and found a series of differentially expressed genes using microarray technology. Based on this, the OPTN-silencing microarray data were re-annotated, differentially expressed lncRNAs were identified and an lncRNA-lncRNA co-expression network was constructed in the present study. As a result, three lncRNAs, namely MMP12, RP11-169D4.1 and RP1-212P9.2 with fold-change values >2 or $<0.5$ were identified. Among them, the first two lncRNAs were upregulated, while the third was downregulated. RT-qPCR assays were performed to verify the results of the re-annotated data and these demonstrated that RP1-212P9.2 was downregulated $(\mathrm{P}<0.01)$, while RP11-169D4.1 was upregulated $(\mathrm{P}<0.01)$, consistent with the re-annotated data.

It is difficult to predict the functions of lncRNAs on the basis of their nucleotide sequences only, since the majority of them are poorly conserved in mammals, as compared with protein-encoding genes (28). Therefore, to explore the potential functions of the lncRNAs in OPTN-silenced cells, a lncRNA/mRNA co-expression network was constructed based on the correlation analysis. This indicated that RP1-212P9.2 and RP11-169D4.1 co-expressed mRNAs are targeted to 'negative regulation of gene expression' and 'microtubule cytoskeleton organization' (ontology, biological process), respectively. These biological processes may be involved with the regulation of gene products, and neuronal migration, polarity and differentiation (29). KEGG pathway analysis indicated that the mRNAs co-expressed with the lncRNAs were involved in biological functions including apoptosis, oxidative phosphorylation, axon guidance, ubiquitin-mediated proteolysis, the cell cycle, lysosome, endocytosis, the TGF- $\beta$ signaling pathway and the neurotropin signaling pathway. These signaling pathways are closely associated with pathological processes such as neurodegeneration $(30,31)$, neuronal cell death, survival and migration (32), suggesting that the lncRNA-mediated network may serve an extensive role in the pathogenesis of OPTN-associated diseases.

Notably, the TGF- $\beta$ signaling pathway and apoptosis pathway are associated with basic biological processes of neural diseases (33-35). The TGF- $\beta$ signaling pathway regulates diverse cellular processes, including cell proliferation, differentiation, plasticity and migration (36). In addition, it is involved in the development of the nervous system and exhibits neuroprotective functions (33). Furthermore, TGF- $\beta$ is a vital growth factor in the pathogenesis of glaucoma (37). It stimulates the proliferation of human Tenon's fibroblasts and alters the cytoskeleton, which causes ocular hypertension and fibronectin deposition in the trabecular meshwork (TM) (38). A previous study indicates that bone morphogenetic proteins (BMPs) antagonize TGF- $\beta$ signaling (39). Furthermore, in glaucomatous TM cells and tissues, a BMP antagonist directly elevated IOP through the canonical TGF- $\beta /$ SMAD pathway (40). In accordance with these studies, the TGF- $\beta$ signaling pathway gained the highest count score during KEGG analysis in the present study.

The apoptosis pathway is a genetically controlled mechanism of cell death involved in the regulation of tissue homeostasis. KEGG analysis in the present study indicated 
A

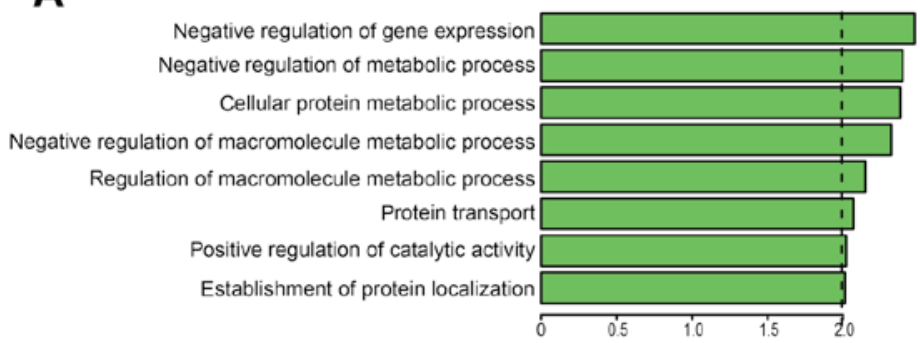

B

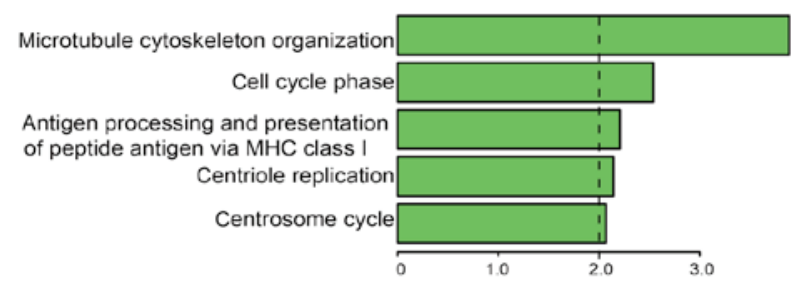

Figure 4. Results of the functional enrichment of two validated long noncoding RNAs by their co-expressed mRNAs. Gene Ontology biological process terms enriched by co-expressed mRNAs of (A) RP1-212P9.2 and (B) RP11-169D4.1 (P<0.01). .
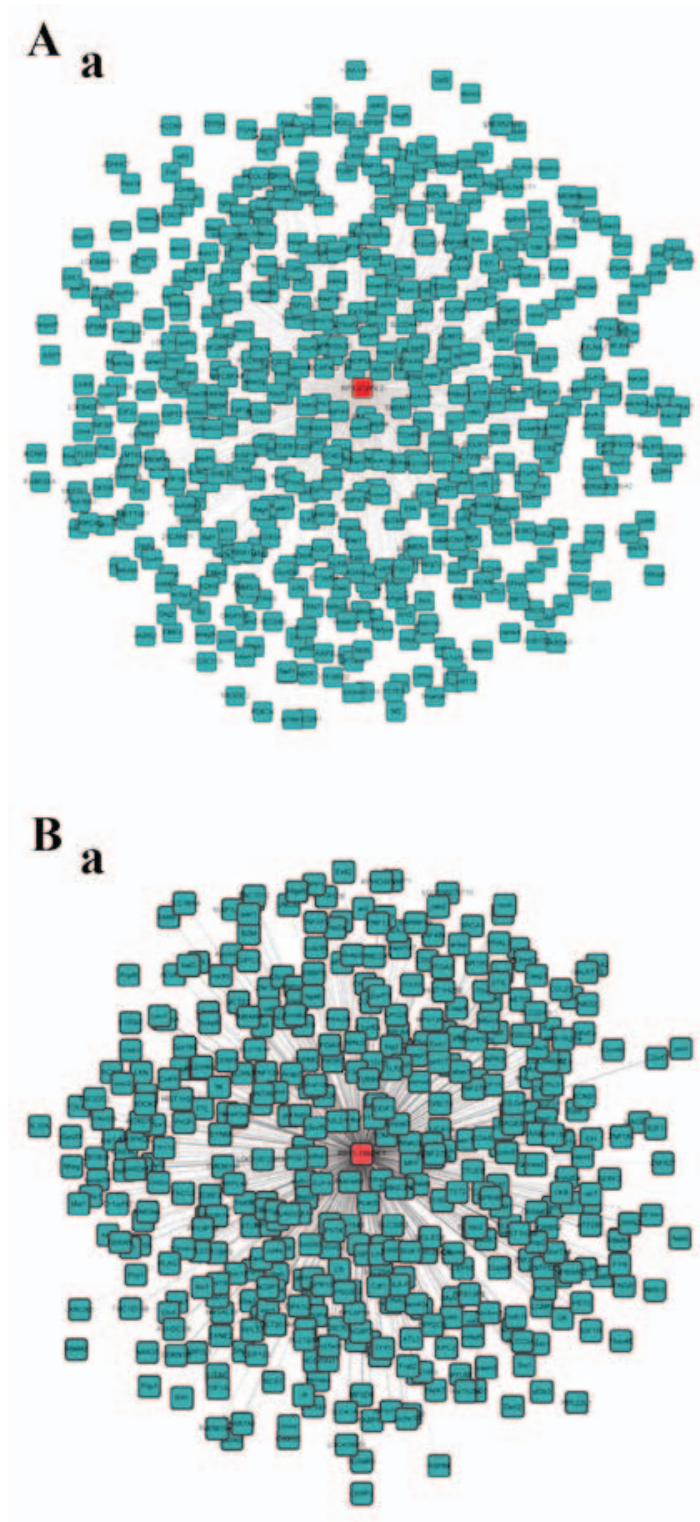

b

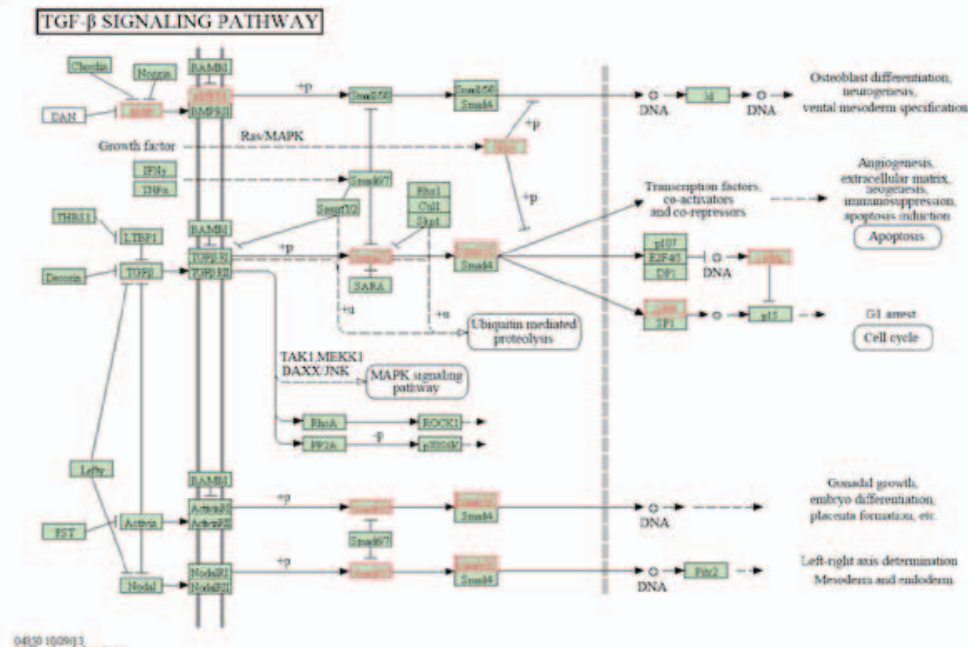

b

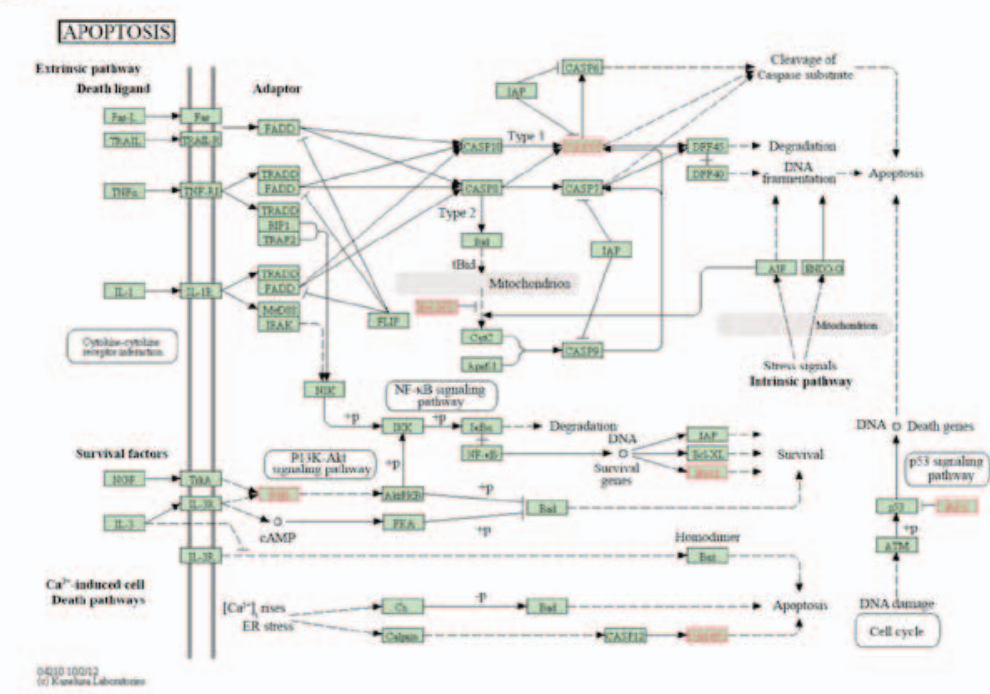

Figure 5. Co-expressed coding genes and pathway analysis of mRNAs co-expressed with long noncoding RNAs. (A-a) Co-expression network for RP1-212P9.2 and its co-expressed mRNAs. (A-b) KEGG analysis suggests that RP1-212P9.2 co-expressed mRNAs are mainly targeted to the TGF- $\beta$ signaling pathway. (B-a) Co-expression network for RP11-169D4.1 and its co-expressed mRNAs. (B-b) KEGG analysis suggests that RP11-169D4.1 co-expressed mRNAs are mainly targeted to the apoptosis signaling pathway. KEGG, Kyoto Encyclopedia of Genes and Genomes; TGF, transforming growth factor.

that RP11-169D4.1 is enriched in apoptosis. This implies that apoptosis is a crucial event in OPTN-silenced cells. POAG is characterized by progressive degeneration induced by the apoptosis of RGCs in optic nerves (41). It has been reported 
that RGC degeneration is partly initiated via apoptotic pathways; among the involved pathways, the phosphoinositide 3-kinase/Akt pathway, Bcl-2 family and caspase family were suggested to be the most important (42). In summary, differentially expressed lncRNAs in OPTN-silenced cells may be crucial in regulating the molecular mechanisms of glaucoma. However, in the future, further exploration of this hypothesis is necessary.

Although the detailed mechanisms of OPTN-associated diseases have not been fully elucidated, the differentially expressed lncRNAs may serve important roles in their corresponding signaling networks, and facilitate the regulation of coding genes. IncRNAs possibly regulate gene expression through directing methylation complexes, initiating chromatin remodeling, targeting transcription factors and blocking nearby transcription (43). The present understanding of the regulatory effect of IncRNA in OPTN mutation is limited. In the future, alternative techniques may be used to determine the biological functions of the lncRNAs, such as lncRNA silencing and structure disruption (44).

In conclusion, the present study demonstrated the ability of functional re-annotation to identify differentially expressed IncRNAs in the microarray dataset. This approach has been successful in the identification of altered lncRNA expression in previous studies $(45,46)$. The present study provides new insights into the involvement of lncRNAs in the pathogenesis of OPTN-associated diseases. However, further study is required to understand the biological functions and molecular mechanisms of the distinct lncRNAs implicated in OPTN-associated diseases.

\section{References}

1. Chi ZL, Akahori M, Obazawa M, Minami M, Noda T, Nakaya N, Tomarev S, Kawase K, Yamamoto T, Noda S, et al: Overexpression of optineurin E50K disrupts Rab8 interaction and leads to a progressive retinal degeneration in mice. Hum Mol Genet 19: 2606-2615, 2010

2. Rezaie T, Child A,Hitchings R, Brice G, Miller L, Coca-Prados M, Héon E, Krupin T, Ritch R, Kreutzer D, et al: Adult-onset primary open-angle glaucoma caused by mutations in optineurin. Science 295: 1077-1079, 2002

3. Allingham RR, Liu Y and Rhee DJ: The genetics of primary open-angle glaucoma: a review. Exp Eye Res 88: 837-844, 2009.

4. Gleason CE, Ordureau A, Gourlay R, Arthur JSC and Cohen P: Polyubiquitin binding to optineurin is required for optimal activation of TANK-binding kinase 1 and production of interferon $\beta$. J Biol Chem 286: 35663-35674, 2011.

5. Hattula K and Peränen J: FIP-2, a coiled-coil protein, links huntingtin to Rab8 and modulates cellular morphogenesis. Curr Biol 10: 1603-1606, 2000.

6. del Toro D, Alberch J, Lázaro-Diéguez F, Martín-Ibáñez R, Xifró X, Egea G and Canals JM: Mutant huntingtin impairs post-Golgi trafficking to lysosomes by delocalizing optineurin/Rab8 complex from the Golgi apparatus. Mol Biol Cell 20: 1478-1492, 2009.

7. Nagabhushana A, Chalasani ML, Jain N, Radha V, Rangaraj N, Balasubramanian D and Swarup G: Regulation of endocytic trafficking of transferrin receptor by optineurin and its impairment by a glaucoma-associated mutant. BMC Cell Biol 11: 4, 2010.

8. Kapranov P,Cheng J,DikeS,Nix DA,DuttaguptaR,Willingham AT, Stadler PF, Hertel J, Hackermüller J, Hofacker IL, et al: RNA maps reveal new RNA classes and a possible function for pervasive transcription. Science 316: 1484-1488, 2007.

9. Carninci P,Kasukawa T, Katayama S, Gough J, Frith MC, Maeda N, Oyama R, Ravasi T, Lenhard B, Wells C, et al; RIKEN Genome Exploration Research Group and Genome Science Group (Genome Network Project Core Group): The transcriptional landscape of the mammalian genome. Science 309: 1559-1563, 2005.
10. Mercer TR, Dinger ME, Sunkin SM, Mehler MF and Mattick JS: Specific expression of long noncoding RNAs in the mouse brain. Proc Natl Acad Sci USA 105: 716-721, 2008.

11. Amaral PP and Mattick JS: Noncoding RNA in development. Mamm Genome 19: 454-492, 2008.

12. Ng SY, Lin L, Soh BS and Stanton LW: Long noncoding RNAs in development and disease of the central nervous system. Trends Genet 29: 461-468, 2013.

13. Johnson R: Long non-coding RNAs in Huntington's disease neurodegeneration. Neurobiol Dis 46: 245-254, 2012.

14. Zhu J, Liu S, Ye F, Shen Y, Tie Y, Zhu J, Jin Y, Zheng X, Wu Y and Fu H: The long noncoding RNA expression profile of hepatocellular carcinoma identified by microarray analysis. PLoS One 9: e101707, 2014.

15. Maruyama $\mathrm{H}$, Morino $\mathrm{H}$, Ito $\mathrm{H}$, Izumi $\mathrm{Y}$, Kato $\mathrm{H}$, Watanabe $\mathrm{Y}$, Kinoshita Y, Kamada M, Nodera H, Suzuki H, et al: Mutations of optineurin in amyotrophic lateral sclerosis. Nature 465: 223-226, 2010

16. Mercer TR, Dinger ME and Mattick JS: Long non-coding RNAs: insights into functions. Nat Rev Genet 10: 155-159, 2009.

17. Moran VA, Perera RJ and Khalil AM: Emerging functional and mechanistic paradigms of mammalian long non-coding RNAs. Nucleic Acids Res 40: 6391-6400, 2012.

18. Weisschuh N, Alavi MV, Bonin M and Wissinger B: Identification of genes that are linked with optineurin expression using a combined RNAi - microarray approach. Exp Eye Res 85: 450-461, 2007.

19. Liao Q, Xiao H, Bu D, Xie C, Miao R, Luo H, Zhao G, Yu K, Zhao H, Skogerbø G, et al: ncFANs: A web server for functional annotation of long non-coding RNAs. Nucleic Acids Res 39: W118-W124, 2011.

20. Langfelder P and Horvath S: WGCNA: An R package for weighted correlation network analysis. BMC Bioinformatics 9: 559,2008

21. Livak KJ and Schmittgen TD: Analysis of relative gene expression data using real-time quantitative PCR and the 2(-Delta Delta C(T)) Method. Methods 25: 402-408, 2001.

22. Huang W, Sherman BT and Lempicki RA: Systematic and integrative analysis of large gene lists using DAVID bioinformatics resources. Nat Protoc 4: 44-57, 2009.

23. Subramanian A, Tamayo P, Mootha VK, Mukherjee S, Ebert BL, Gillette MA, Paulovich A, Pomeroy SL, Golub TR, Lander ES, et al: Gene set enrichment analysis: A knowledge-based approach for interpreting genome-wide expression profiles. Proc Natl Acad Sci USA 102: 15545-15550, 2005.

24. Schlitt T, Palin K, Rung J, Dietmann S, Lappe M, Ukkonen E and Brazma A: From gene networks to gene function. Genome Res 13: 2568-2576, 2003

25. Yan B, Tao ZF, Li XM, Zhang H, Yao J and Jiang Q: Aberrant expression of long noncoding RNAs in early diabetic retinopathy. Invest Ophthalmol Vis Sci 55: 941-951, 2014.

26. Albagha OM, Visconti MR, Alonso N, Langston AL, Cundy T, Dargie R, Dunlop MG, Fraser WD, Hooper MJ, Isaia G, et al: Genome-wide association study identifies variants at $C S F 1$, OPTN and TNFRSF11A as genetic risk factors for Paget's disease of bone. Nat Genet 42: 520-524, 2010.

27. Soto I, Oglesby E, Buckingham BP, Son JL, Roberson ED, Steele MR, Inman DM, Vetter ML, Horner PJ and Marsh-Armstrong N: Retinal ganglion cells downregulate gene expression and lose their axons within the optic nerve head in a mouse glaucoma model. J Neurosci 28: 548-561, 2008.

28. Chodroff RA, Goodstadt L, Sirey TM, Oliver PL, Davies KE, Green ED, Molnár Z and Ponting CP: Long noncoding RNA genes: conservation of sequence and brain expression among diverse amniotes. Genome Biol 11: R72, 2010.

29. Kapitein LC and Hoogenraad CC: Building the neuronal microtubule cytoskeleton. Neuron 87: 492-506, 2015.

30. Espinet C, Gonzalo H, Fleitas C, Menal MJ and Egea J: Oxidative stress and neurodegenerative diseases: A neurotrophic approach. Curr Drug Targets 16: 20-30, 2015.

31. Wakatsuki S, Furuno A, Ohshima M and Araki T: Oxidative stress-dependent phosphorylation activates ZNRF1 to induce neuronal/axonal degeneration. J Cell Biol 211: 881-896, 2015.

32. Herrup K, Neve R, Ackerman SL and Copani A: Divide and die: Cell cycle events as triggers of nerve cell death. J Neurosci 24: 9232-9239, 2004.

33. Dobolyi A, Vincze C, Pál G and Lovas G: The neuroprotective functions of transforming growth factor beta proteins. Int J Mol Sci 13: 8219-8258, 2012. 
34. Wang SP, Wang ZH, Peng DY, Li SM, Wang H and Wang XH Therapeutic effect of mesenchymal stem cells in rats with intracerebral hemorrhage: Reduced apoptosis and enhanced neuroprotection. Mol Med Rep 6: 848-854, 2012.

35. Liu G, Wang X, Shao G and Liu Q: Genetically modified Schwann cells producing glial cell line-derived neurotrophic factor inhibit neuronal apoptosis in rat spinal cord injury. Mol Med Rep 9: 1305-1312, 2014.

36. Zhao B and Chen YG: Regulation of TGF- $\beta$ signal transduction. Scientifica (Cairo) 2014: 874065, 2014.

37. Wordinger RJ, Clark AF, Agarwal R, Lambert W and Wilson SE: Expression of alternatively spliced growth factor receptor isoforms in the human trabecular meshwork. Invest Ophthalmol Vis Sci 40: 242-247, 1999.

38. McDowell CM, Tebow HE, Wordinger RJ and Clark AF: Smad3 is necessary for transforming growth factor-beta2 induced ocular hypertension in mice. Exp Eye Res 116: 419-423, 2013.

39. Zode GS, Clark AF and Wordinger RJ: Bone morphogenetic protein 4 inhibits TGF- $\beta 2$ stimulation of extracellular matrix proteins in optic nerve head cells: role of gremlin in ECM modulation. Glia 57: 755-766, 2009.
40. Sethi A, Wordinger RJ and Clark AF: Gremlin utilizes canonical and non-canonical TGF $\beta$ signaling to induce lysyl oxidase (LOX) genes in human trabecular meshwork cells. Exp Eye Res 113: 117-127, 2013.

41. Kuehn MH, Fingert JH and Kwon YH: Retinal ganglion cell death in glaucoma: mechanisms and neuroprotective strategies. Development 1: 3, 2005.

42. Levkovitch-Verbin H: Retinal ganglion cell apoptotic pathway in glaucoma: Initiating and downstream mechanisms. Prog Brain Res 220: 37-57, 2015.

43. Ponting CP, Oliver PL and Reik W: Evolution and functions of long noncoding RNAs. Cell 136: 629-641, 2009.

44. Sánchez Y and Huarte M: Long non-coding RNAs: Challenges for diagnosis and therapies. Nucleic Acid Ther 23: 15-20, 2013.

45. Gao W, Chan JY and Wong TS: Differential expression of long noncoding RNA in primary and recurrent nasopharyngeal carcinoma. Biomed Res Int 2014: 404567, 2014.

46. Michelhaugh SK, Lipovich L, Blythe J, Jia H, Kapatos G and Bannon MJ: Mining Affymetrix microarray data for long non-coding RNAs: Altered expression in the nucleus accumbens of heroin abusers. J Neurochem 116: 459-466, 2011. 